\title{
Sildenafil Slows Gastric Emptying in Patients with Previously Diagnosed Diabetic Gastroparesis
}

\author{
Imaneh Fallahi ${ }^{1}$, Susannah T Clark ${ }^{2}$, Suzanne Emil ${ }^{1}$, Robert J Telepak ${ }^{1}$ and Mark R Burge ${ }^{1 *}$ \\ ${ }^{1}$ Department of Internal Medicine, Endocrinology \& Metabolism, University of New Mexico Health Sciences Center, USA \\ ${ }_{2}^{2}$ Internal Medicine, Geriatrics, Alice Peck Day Memorial Hospital, Lebanon
}

*Corresponding author: Mark R Burge, University of New Mexico Health Sciences Center, Department of Internal Medicine, Endocrinology \& Metabolism, New Mexico, USA.

To Cite This Article: Imaneh Fallahi, Susannah T Clark, Suzanne Emil, Robert J Telepak, Mark R Burge, Sildenafil Slows Gastric Emptying in Patients with Previously Diagnosed Diabetic Gastroparesis. Am J Biomed Sci \& Res. 2020 - 11(3). AJBSR.MS.ID.001637.

DOI: 10.34297/AJBSR.2020.11.001637.

Received: 眥 December 09, 2020; Published: 眥 December 21, 2020

\begin{abstract}
Objective: Delayed gastric emptying is a common complication of diabetes. Animal studies suggest that inhibition of phosphodiesterase type5 receptors with sildenafil improves pyloric nitric oxide availability and reverses gastroparesis in murine models of diabetic gastroparesis. We hypothesized that a single dose of sildenafil would improve gastric emptying in diabetic subjects with gastroparesis.

Design: Thirteen subjects with diabetes and gastroparesis were admitted to the UNM General Clinical Research Center on two occasions at least one week apart. Gastric motility agents were withheld 48 hours prior to study. Overnight glycemia was stabilized with infusion of intravenous insulin. At 0800 , subjects ingested either $50 \mathrm{mg}$ of sildenafil or matching placebo in a randomized, double blind, crossover protocol. Gastric emptying was determined using radionuclide scintigraphy following an egg and toast meal with 99Tc-sulfur colloid at 0900 on each study morning.

Results: Fasting glucose concentrations did not differ between the two study conditions: placebo $=114 \pm 31 \mathrm{vs}$. sildenafil $=111 \pm 53 \mathrm{mg} / \mathrm{dl}$, $\mathrm{p}=0.81$. The half-time for gastric emptying as determined by radionuclide scintigraphy was increased after administration of sildenafil $(328 \pm 301$ minute) compared to placebo ( $163 \pm 113$ minutes; $\mathrm{p}=0.03$ ).

Conclusion: This study demonstrates that the administration of a single oral dose of sildenafil further slows gastric emptying in patients with diabetic gastroparesis. These data suggest that phosphodiesterase type-5 inhibitors are not effective for the treatment of diabetic gastroparesis.
\end{abstract}

Keywords: Diabetic Gastroparesis; Sildenafil; Therapeutics; Mechanisms of Disease

\section{Significance of the Study}

What is already known about this subject?

1. Pyloric function is regulated by nitric oxide availability.

2. Nitric oxide is produced in the pylorus by the enzyme phosphodiesterase type 5 .

3. Sildenafil potently inhibits phosphodiesterase type 5 .

What are the new findings?
1. Pre-meal sildenafil slowed gastric emptying as compared to placebo in patients with diabetic gastroparesis.

How might these results change the focus of research or clinical practice?

1. Targeting the inhibition phosphodiesterase type 5 for the treatment of diabetic gastroparesis does not appear to be an effective strategy. 


\section{Contributorship}

IF salvaged forgotten data and re-analyzed study data and prepared the manuscript. STC and NE collected, recorded, and analyzed study data. RJT performed the gastric emptying studies. MRB designed the study, secured funding, obtained regulatory approvals, oversaw protocol implementation, and finalized the manuscript.

\section{Introduction}

Delayed gastric emptying is a common complication of longstanding diabetes mellitus, with $50-75 \%$ of all patients exhibiting some degree of gastrointestinal neuropathy and/or delayed gastric emptying [1]. When present, symptoms include postprandial abdominal distention, bloating, pain, nausea, and vomiting, anorexia and early satiety. Gastroparesis can occur both in insulin dependent and non-insulin dependent diabetes patients [2]. This syndrome contributes to the morbidity of diabetes because severe hyperglycemia and hypoglycemia frequently result from an inability to precisely match the action of pharmacotherapeutics such as injected insulin with the absorption of carbohydrate from the gut $[3,4]$. As a result, target blood glucose goals are difficult to achieve in affected patients. Assessment of gastric emptying time using radionuclide scintigraphy with 99Tc sulphur colloid labeling of a solid test meal is the current clinical standard for the diagnosis and monitoring of this condition $[5,6]$.

Nitric oxide (N0) is a gas that serves as a neurotransmitter and paracrine hormonal agent in humans, and it is involved in the control of numerous vascular and gastrointestinal processes. Specific functions of nitric oxide in the human gastrointestinal tract include esophageal relaxation, stomach relaxation, pyloric relaxation, enhancement of gastric mucosal blood flow, and enhancement intestinal secretion [7]. In the human gastrointestinal tract, relaxation of smooth muscle in the proximal stomach is mediated through release of NO from non-adrenergic, noncholinergic inhibitory neurons in the gastric wall. Nitric oxide diffuses through the cell membrane of the smooth muscle cells, where it increase the concentration of cyclic guanylyl monophosphate (cGMP) and thus initiates a process that ends in hyperpolarization. As a result, the smooth muscle of the proximal stomach relaxes to keep intra-gastric pressure low while intra-gastric volume increases. Progressive filling of the proximal stomach induces a rise in intragastric pressure, followed by redistribution of gastric contents from the proximal stomach to the antrum, allowing the initiation of gastric emptying [8]. Nitric oxide is also released by nonadrenergic, noncholinergic inhibitory neurons in the esophagus and has been shown to cause relaxation of the lower esophageal sphincter and to regulate peristaltic contractions through a cGMPmediated mechanism $[9,10]$.
There is mounting evidence in animals that diabetes causes a reversible down regulation of neuronal nitric oxidase synthase (n-NOS) which results in delayed gastric emptying due to impaired pyloric relaxation [11]. Furthermore, knockout animals deficient in n-NOS exhibit gastrointestinal dysmotility that is very similar to that which occurs in diabetic gastroparesis [11]. One report demonstrated that sildenafil administration reversed impaired stomach emptying in both NOD and STZ diabetic mice with gastroparesis [11]. Sildenafil is a potent inhibitor of phosphodiesterase type-5 (PDE-5) that produces a clinical effect by increasing the signal generated by a given amount of nitric oxide, thereby attenuating the effect of reduced nitric oxide generation. Moreover, PDE-5 has been specifically localized in the human pylorus as well as in erectile tissue [12]. Similarly, the esophageal dysmotility observed in achalasia has been shown to be attributable to a depletion of n-NOS [10].

The current gold standard for the clinical assessment of gastric emptying is measurement of the scintigraphic disappearance of a radionuclide (99Tc)-labeled test meal from the stomach [13]. This study assesses whether or not sildenafil therapy improves gastric emptying time as determined by a 99Tc sulphur colloid solid meal gastric emptying study in patients with previously diagnosed diabetic gastroparesis. We hypothesized that inadequate nitric oxide generation and/or stability plays a role in the pathogenesis of diabetic gastroparesis in humans and that this defect is amenable to pharmacologic intervention with agents that prolong nitric oxide action and/or stability, such as sildenafil.

\section{Research Design and Methods}

We performed a randomized, double blind, placebo controlled, cross over clinical trial of the effect of sildenafil on gastric emptying in patients with diabetic gastroparesis. We hypothesized that 50 mg of sildenafil taken 60 minutes prior to ingestion of a standard radiolabeled test meal would improve gastric emptying time as determined by radionuclide scintigraphy compared to placebo.

Thirteen adult male and female subjects with diabetes mellitus who had been previously diagnosed with diabetic gastroparesis within the past five years were enrolled into the study during 20022003. All subjects rendered informed consent as approved by the University of New Mexico Human Research Review Committee. Of note, this study was designed, performed and completed prior to the legal requirements for the registration of clinical trials with clinicaltrials.gov in 2005, but the study was performed under FDA oversight (IND \# 65,046).

Study inclusion criteria included an established diagnosis of type 1 or 2 diabetes for at least one year, diabetic gastroparesis diagnosed during the past 5 years with a $\mathrm{T} 1 / 2$ for gastric emptying greater than 90 minutes, and age between 21 and 75 years, 
inclusive. Criteria for patient exclusion included a history of New York Heart Association Class III or IV cardiovascular disease or lifethreatening arrhythmia, use of drugs other than prescribed gastric motility agents that are known to alter gastric motility within the past 30 days (i.e.- glucagon, octreotide, domperidone), underlying renal or hepatic insufficiency, a history of anatomic abnormalities of the upper gastrointestinal tract, malignancy, known sensitivity to sildenafil or a contraindication to sildenafil therapy, active substance abuse or alcoholism, pregnancy or lactation, resting hypotension (i.e.- BP < 90/50 $\mathrm{mmHg}$ ), a history of retinitis pigmentosa, and/or inability to give informed consent.

After a screening evaluation, all enrolled subjects participated in two overnight studies at least one week apart in random order. Subjects were admitted to the University of New Mexico General Clinical Research Center on the afternoon prior to each study. Gastric motility agents such as metoclopramide and erythromycin were withheld for at least 48 hours prior to study. All subjects received a standard $10 \mathrm{Kcal} / \mathrm{kg}$ meal in accordance with the recommendations of the American Diabetes Association at 1800 on the day of admission. Food was subsequently withheld until completion of the study, which was performed at 0800 the following morning after 14 hours of fasting. All patients were allowed free access to one liter of water or non-caloric, noncaffeinated beverages during the overnight study.

Because gastric motility can vary with ambient glucose concentrations, all subjects had their blood glucose concentrations controlled overnight with a continuous infusion of intravenous insulin to maintain euglycemia (i.e.- $80-120 \mathrm{mg} / \mathrm{dl}$ ) between 1800 and 0800 the following morning, and the rate of insulin infusion was adjusted overnight to maintain target glucose levels during the insulin infusion [14-17]. At 0800 the following morning, subjects ingested a single $50 \mathrm{mg}$ oral dose of sildenafil or matching placebo (Pfizer Inc, Groton, CT). At 0900, subjects ingested a standardized egg and toast breakfast that included $1 \mathrm{mCi}$ of 99Tc sulphur colloid. A standard gastric emptying study was subsequently performed and a $\mathrm{T}^{1} 1 / 2$ for gastric emptying was determined as previously described [6]. An identical study was performed at least one week later using whichever study medication was not used during the first study (i.e.- sildenafil or placebo). Sildenafil is rapidly absorbed from the gastrointestinal tract, with peak blood concentrations occurring 30 to 120 minutes following ingestion [18]. Thus, peak blood levels should have been well matched to peak gastric emptying activity in this study. After 0800, intravenous insulin doses were fixed at the minimal level necessary to maintain target blood glucose levels prior to ingestion of the test meal during the gastric emptying study.

The primary study end point for this study was the half-time $\left(\mathrm{T}^{1} 1 / 2\right)$ for gastric emptying as determined by radionuclide scintigraphy. A
$\mathrm{T} 1 / 2$ greater than 90 minutes is diagnostic of diabetic gastroparesis [6]. Secondary study endpoints included fasting plasma glucose and esophageal transit time (or "swallowing time"). Esophageal transit time was determined using a stopwatch and a stethoscope placed over the epigastrium. Subjects were asked to swallow $30 \mathrm{ml}$ of water while sitting at $45^{\circ}$, and the time between ingesting the water and the audible sound associated with the opening of the lower esophageal sphincter was recorded as the esophageal transit time. For the purposes of sample size determination, clinical data were used to determine the mean and standard deviation $\mathrm{T} 1 / 2$ for gastric emptying time in patients with diabetic gastroparesis at our institution $(157 \pm 75$ minutes). When using a paired Student's t-test to compare the T1/2 for gastric emptying between the two study conditions, twelve subjects studied under each condition will provide $80 \%$ power to detect a difference in gastric emptying time of 45 minutes with alpha equal to 0.05 .

The order of randomization (sildenafil first or placebo first) was determined using a random number generator, and the randomization list was maintained by the investigational pharmacist at the University of New Mexico Hospital.

All available study data were included in the analysis. Data were compared between the sildenafil and placebo studies using the paired Student's t-test. Data are reported as mean \pm standard deviation.

\section{Results}

Baseline and descriptive characteristics of the study participants are summarized in Table 1 . The thirteen subjects (9 females and 4 males) were aged $39 \pm 13$ years, and eight had type 1 diabetes mellitus while five had type 2 diabetes. The mean duration of diabetes was $17 \pm 3$ years, baseline A1c levels were $8.9 \pm 2.5 \%$, mean baseline BMI was $27.7 \pm 6.5 \mathrm{~kg} / \mathrm{m} 2$, and baseline $\mathrm{T} 1 / 2$ for gastric emptying was $364 \pm 498$ minutes. All participants completed both study arms.

Figure 1A displays fasting glucose concentrations between the two study conditions. Fasting glucose concentrations did not differ significantly between the two study conditions (placebo $=114 \pm 31$ $\mathrm{mg} / \mathrm{dl}$ versus sildenafil $=111 \pm 53 \mathrm{mg} / \mathrm{dl} ; \mathrm{p}=0.81$ ). Esophageal emptying times for the two study conditions are shown in Figure 1B. There was no significant difference between the two study conditions with respect to this parameter (placebo $=5.9 \pm 5.8$ seconds versus sildenafil $=3.6 \pm 2.2$ seconds; $p=0.30$ ). Half-times for gastric emptying for the two study conditions are summarized in Figure 1 . The $\mathrm{T}^{1} \frac{1}{2}$ for gastric emptying was significantly increased following administration of sildenafil compared to administration of placebo (placebo $=163 \pm 113$ minutes versus sildenafil $=328 \pm 301$ minutes; $\mathrm{p}=0.03$ ). 


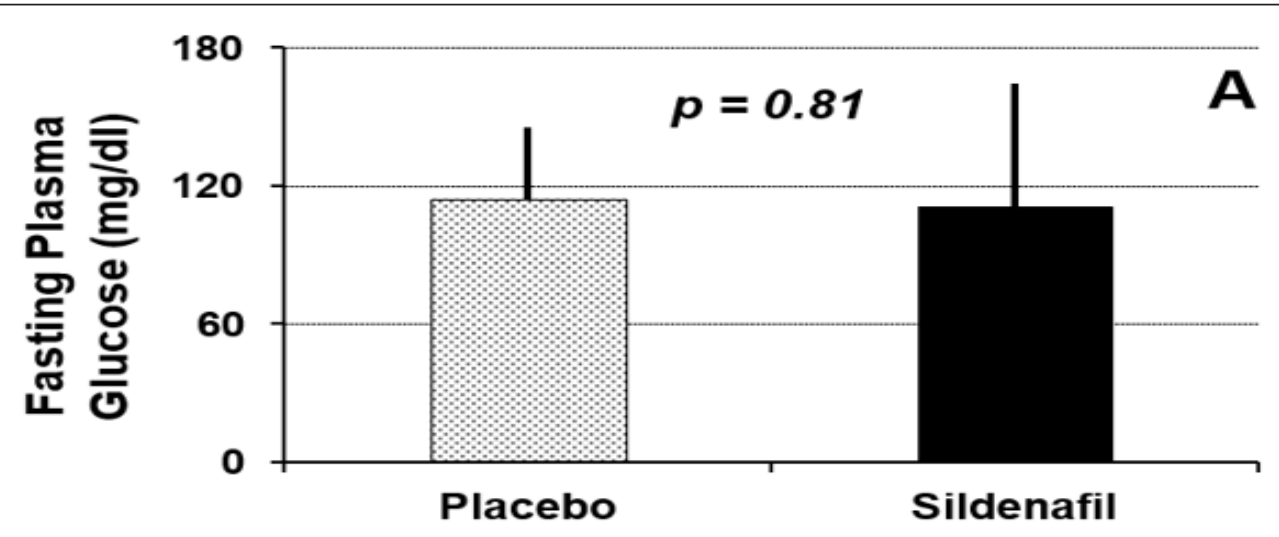

Figure 1A: Fasting glucose concentration among 13 subjects with diabetic gastroparesis randomized to receive sildenafil $50 \mathrm{mg}$ po (solid bars) or matching placebo (stippled bars).

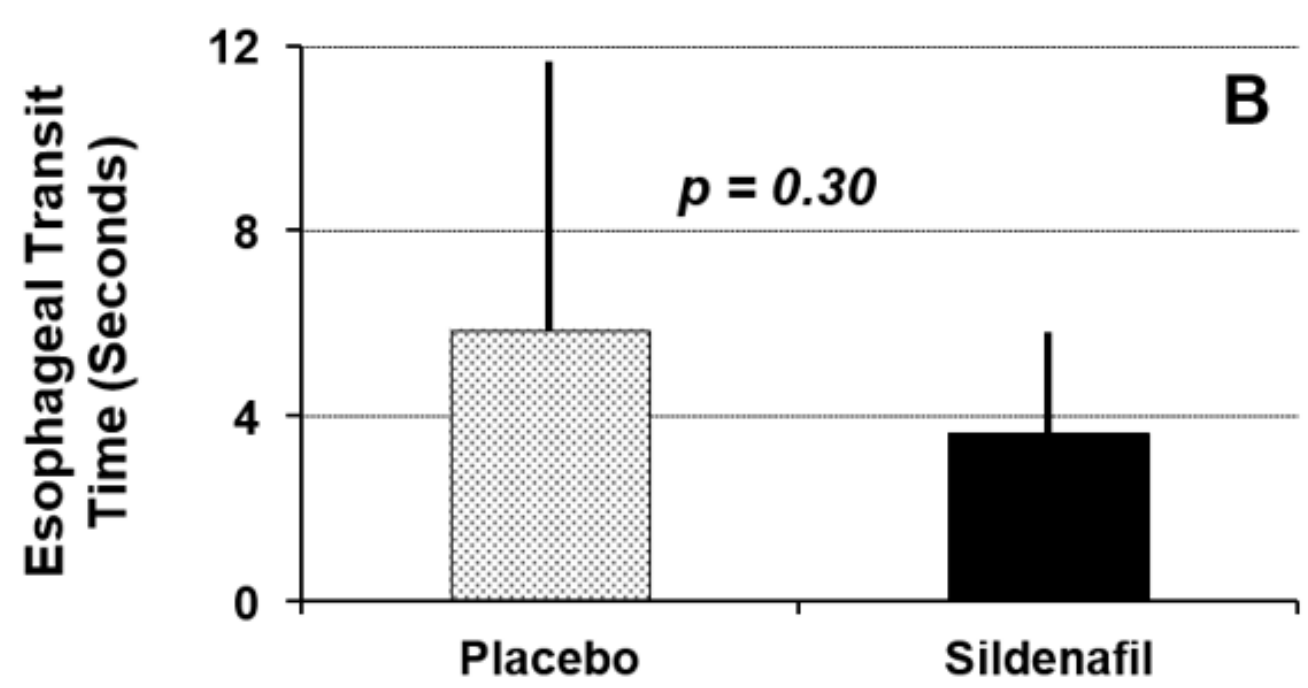

Figure 1B: Esophageal transit time for liquids among 13 subjects with diabetic gastroparesis randomized to receive sildenafil $50 \mathrm{mg}$ po or matching placebo.

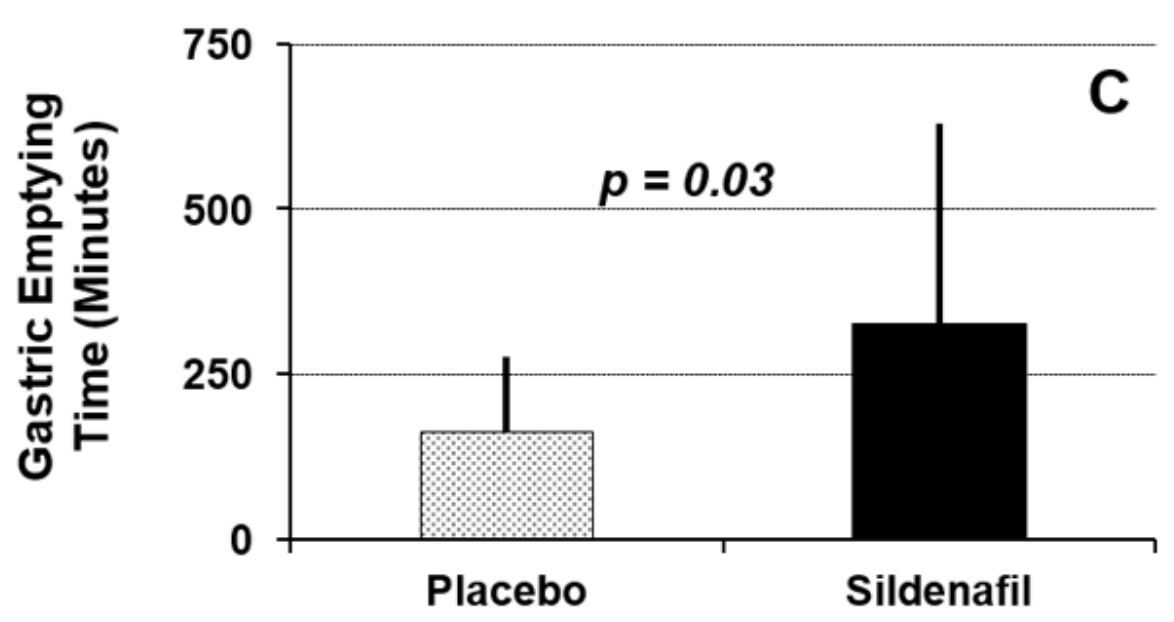

Figure 1C: Gastric emptying time as determined by 99Tc radionuclide testing following a standard solid meal among 13 subjects with diabetic gastroparesis randomized to receive sildenafil $50 \mathrm{mg}$ orally or matching placebo. 
Table 1: Baseline and descriptive characteristics of study participants.

\begin{tabular}{|c|c|}
\hline Characteristic & Mean \pm Std. Dev. \\
\hline Age (years) & $39 \pm 13$ \\
\hline Sex & $4 \mathrm{M}, 9 \mathrm{~F}$ \\
\hline Duration of Diabetes (years) & $17 \pm 3$ \\
\hline Type of Diabetes & 8 Type 1,5 Type 2 \\
\hline $\mathrm{A}_{1 \mathrm{C}}(\%)$ & $8.9 \pm 2.5$ \\
\hline Body Mass Index $\left(\mathrm{kg} / \mathrm{m}^{2}\right)$ & $27.7 \pm 6.5$ \\
\hline Baseline $\mathrm{T}_{1 / 2}$ for Gastric Emptying (minutes) & $364 \pm 498$ \\
\hline
\end{tabular}

\section{Discussion}

This study demonstrates that the administration of a single oral dose of sildenafil slows gastric emptying in patients with established diabetic gastroparesis. This finding was contrary to our hypothesis but is consistent with the results of the study published by Dishy and colleagues, which showed that sildenafil did not improve gastric emptying in patients with ends stage renal failure and gastroparesis. In that study, it was postulated that sildenafil may have had inhibitory effects on gastric peristalsis, causing gastric relaxation in conjunction with its effects on pyloric relaxation [19]. A study by Carbone and colleagues demonstrated that sildenafil inhibited gastric accommodation among healthy volunteers, leading to significantly decreased nutrient tolerance and slight delays in the gastric emptying rate [20]. A study in mice by Patil et al. [21] showed that sildenafil at doses of $0.5-2 \mathrm{mg} / \mathrm{kg}$ did not alter gastric emptying times but that sildenafil in higher doses (5-30 mg/kg) inhibited the gastric emptying [21].

Piswanger-Solkner and colleagues published an abstract detailing the preliminary results of an investigation comparing the effects of $50 \mathrm{mg}$ of sildenafil on gastric emptying in 10 subjects with longstanding diabetes mellitus that were consistent with our study. The subjects had gastric emptying studies performed before and after sildenafil administration, and the results demonstrated that at 140 minutes, gastric emptying was delayed in those subjects who received sildenafil [22]. This study did not, however, control for plasma glucose concentrations, and it is well established that prevailing glycemia can affect the results of gastric emptying studies [23]. In this study, patients were selected based upon the duration of their diabetes and they did not have a previously established diagnosis of diabetic gastroparesis.

In a case report, Bianco and colleagues reported that $50 \mathrm{mg}$ of sildenafil improved gastric lag time, gastric emptying time, and residual retained activity in two human female patients with type 1 diabetes mellitus and refractory diabetic gastropathy as evidenced by improvement in baseline gastric emptying studies [24]. However, this study did not have enough power to be applicable in the clinical setting. Eherer and colleagues reported that sildenafil decreased lower esophageal sphincter tone and reduced propulsive forces in the body of the esophagus in healthy subjects and in patients with nutcracker esophagus or hypertensive lower esophageal sphincter and achalasia [9]. Direct comparison of the current study with the work of other investigators is limited by differences in design, subject characteristics and the small number of available studies. The ineffectiveness of sildenafil in improving gastric emptying times in diabetes patients could be explained by the underlying the mechanism of action of sildenafil. Specifically, although NO may enhance pyloric relaxation, it may also cause simultaneous gastric relaxation, resulting in global gastric stasis and prolongation of gastric emptying time. By augmenting NO effects in the stomach, sildenafil may result in increased fundic relaxation, which may offset its effects on pyloric relaxation so that no significant increase in overall gastric emptying rate occurs [19]. Our study did not incorporate direct measurement of intraesophageal or gastric pressures. Alternatively, neuropathic damage to the intrinsic neurons of the esophagus and/or the stomach may impair the production of NO and cGMP, rendering the effects of PDE-5 inhibition by sildenafil moot and ineffective [10].

Potential limitations of the current study include the lack of long-term sildenafil administration, lack of manometry or symptom data, lack of drug levels to demonstrate that the drug was adequately absorbed, and the familiar difficulty of matching drug absorption with food ingestion in gastroparesis. In addition, patients were predominantly female and the study was done in a single center research setting. Strengths of the current study include its double blind, placebo-controlled design, the fact that study drug administration was observed by health care professionals, and that glycaemia between the study conditions was controlled. In addition, there was careful matching of the gastric emptying study with the anticipated peak activity of orally administered sildenafil.

In summary, these results demonstrate that a single dose of sildenafil slows gastric emptying in subjects with previously established diabetic gastroparesis. Currently, the pharmacologic treatment of diabetic gastroparesis using readily available, FDA 
approved drugs is limited to medications such as metoclopramide in the United States. Because metoclopramide therapy is limited by CNS side effects and the development of tardive dyskinesia, the identification of other effective treatment options is imperative. These results strongly suggest that phosphodiesterase type 5 inhibitors are not likely to prove to be a therapeutic breakthrough for the treatment of diabetic gastroparesis.

\section{Acknowledgment}

This research was supported by the University of New Mexico General Clinical Research Center(NIH NCRR \# 5 M01-RR00997) and by the UNM Clinical \& Translational Science Center (NIHNCATS \#8UL1TR000041). Study drug and placebo were kindly provided by Pfizer, Inc.Thisresearch was performed under FDA IND \# 65,046.

\section{References}

1. Kassander P (1958) Asymptomatic gastric retention in diabetes. Ann Intern Med 48(4): 797-812.

2. Patterson D, Abell T, Rothstein R, Koch K, Barnett J (1999) A doubleblind multicenter comparison of domperidone and metoclopramide in the treatment of diabetic patients with symptoms of gastroparesis. Am J Gastroenterol 94(5): 1230-1234.

3. Fraser RJ, Horowitz M, Maddox AF, Harding PE, Chatterton BE, et al. (1990) Hyperglycaemia slows gastric emptying in type 1 (insulin dependent) diabetes mellitus. Diabetologia 33: 675-680.

4. Varis K (1991) Diabetic gastroparesis: A review. J Diab Comp 5: 207-217.

5. Loo FD, Palmer DW, Soergel KH, Kalbfleisch JH, Wood CM (1984) Gastric emptying in patients with diabetes mellitus. Gastroenterology 86 (3): 485-494.

6. Mettler FA (1998) Imaging of the Gastrointestinal Tract. In: Mettler FA, et al. (Eds.) Essentials of nuclear medicine imaging (Fourth Edition), Guiberteau MJ, WB Saunders, Philadelphia, pp. 237-281.

7. Salzman AL (1995) Nitric oxide in the gut. New Horizons 3(1): 33-45.

8. Surkes HK (2007) cGMP-dependent protein kinase I and smooth muscle relaxation: a tale of two isoforms. Circulation research 101(11): 10781080.

9. Eherer AJ, Schwetz I, Hammer HF, Petnehazy T, Scheidl SJ, et al. (2002) Effect of sildenafil on oesophgeal motor function in healthy subjects and patients with oesophageal motor disorders. Gut 50(6): 758-764.

10. Bortolotti M, Mari C, Lopilato C, Porrazzo G, Miglioli M (2000) Effects of sildenafil on esophageal motility of patients with idiopathic achalasia. Gastroenterology 118(2): 253-257.
11. Watkins CC, Sawa A, Jaffrey S, Blackshaw S, Barrow RK, et al. (2000) Insulin restores neuronal nitric oxides synthase expression and function that is lost in diabetic gastropathy. J Clin Invest 106(3): 373-384.

12. Kotera J, Fujishige K, Akatsuka H, Imai Y, Yanaka N, et al. (1998) Novel alternative splice variants of cGMP-binding cGMP-specific phosphodiesterase. J Biol Chem 273(41): 26982-269890.

13. Burge MR, Tuttle MS, Violett JL, Stephenson CL, Schade DS (2000) Potato-lactulose breath hydrogen testing as a function of gastric motility in diabetes mellitus. Diabetes Technology and Therapeutics 2(2): 241248.

14. Burge MR, Garcia N, Qualls CR, Schade DS (2001) Differential effects of fasting and dehydration in the pathogenesis of diabetic ketoacidosis. Metabolism 50(2): 171-177.

15. Schvarcz E, Palmer M, Aman J, Berne C (1997) Accelerated gastric emptying during hypoglycaemia is not associated with changes in plasma motilin levels. Acta Diabetol 34(3): 194-198.

16. Kong MF, Horowitz M (1999) Gastric emptying in diabetes mellitus: relationship to blood-glucose control. Clin Geriatr Med 15(2): 321-338.

17. Bjornsson ES, Urbanavicius V, Eliasson B, Attvall S, Smith U, et al. (1994) Effects of hyperglycemia in interdigestive gastrointestinal motility in humans. Scand J Gastroenterol 29(12): 1096-1104.

18. (2000) Anonymous. Sildenafil. In: Montvale NJ, et al. (Eds.) Physician's Desk Reference, Medical Economics Press, pp. 2381-2384.

19. Dishy V, Cohen Pour M, Feldman L, Naftali T, Baumer M, et al. (2004) The effect of sildenafil on gastric emptying in patients with end-stage renal failure and symptoms of gastroparesis. Clinical pharmacology and therapeutic 76(3): 281-286.

20. Carbone F, Tack J (2018) The effect of sildenafil on gastric motility and satiation in healthy controls. United European Gastroenterology Journal 6(6): 846-854.

21. Patil CS, Singh VP, Jain NK, Kulkarni SK (2005) Inhibitory effect of sildenafil on gastrointestinal smooth muscle: role of NO-cGMP transduction pathway. Indian Journal of Experimental Biology 43(2): 167-171.

22. Piswanger-Solkner JC, Koch H, Schnedl WJ, Hammer HF, Lipp RW (2002) Influence of sildenafil on gastric emptying in longstanding type 1 diabetes. Eur J Nuclear Med Molec Imaging 29(Suppl. 1): 403.

23. Van Petersen AS, Vu MK, Lam WF, Lamers CB, Ringers J, et al. (2000) Effects of hyperglycaemia and hyperinsulinaemia on proximal gastric motor and sensory function in humans. Clin Sci 99(1): 37-46.

24. Bianco A, Pitocco D, Valenza V, Caputo S, Grieco A, et al. (2002) Effect of Sildenafil on Diabetic Gastropathy. Diabetes Care 25(10): 1888-1889. 